\title{
Relación entre la disponibilidad alimentaria y la mortalidad por cáncer colorrectal en América
}

\author{
Association between food availability and mortality due \\ to colorectal cancer in the Americas
}

'Licenciada en Nutrición, Magíster en Epidemiología, Gestión y Políticas de Salud. Docente adscripta, Universidad de Buenos Aires, Ciudad autónoma de Buenos Aires, Argentina. $\triangle$ (iD)
RESUMEN Con el objetivo de describir la relación entre la disponibilidad alimentaria y la tasa de mortalidad por cáncer colorrectal en los países de América en el año 2010, se analizaron datos provistos por la International Agency for Research on Cancer y la Food and Agriculture Organization mediante un estudio ecológico. Se observó una gran variabilidad excepto en disponibilidad calórica. La disponibilidad alimentaria fue abundante para calorías, grasas totales, grasa animal, carnes rojas y bebidas alcohólicas. Para frutas y vegetales fue crítica en un $80 \%$ de los países. Los países con más alta tasa de mortalidad por cáncer colorrectal fueron Uruguay, Barbados, Argentina y Cuba, y con las tasas más bajas fueron Guatemala, Canadá, México y Honduras. Las relaciones más fuertes se dieron entre la tasa de mortalidad por cáncer colorrectal y la disponibilidad alimentaria de grasa animal, carne roja, bebidas alcohólicas y calorías. No se encontró efecto protector de la disponibilidad alimentaria de frutas y vegetales sobre la tasa de mortalidad por cáncer colorrectal. Sería recomendable mejorar los registros de incidencia de tumores y de formas directas de evaluar la dieta para ser analizados en futuros estudios en lugar de los datos aquí utilizados.

PALABRAS CLAVES Neoplasias Colorrectales; Mortalidad; Dieta; Abastecimiento de Alimentos; Factor de Riesgo.

\begin{abstract}
With the aim of describing the association between food availability and the mortality rate due to colorectal cancer in the countries of the Americas in 2010, data provided by the International Agency for Research on Cancer and the Food and Agriculture Federation were analyzed in an ecological study. Great variability was observed except in caloric supply. Food availability was abundant for calories, total fats, animal fat, red meat and alcoholic beverages. Availability was critically low for fruits and vegetables in $80 \%$ of the countries. The countries with the highest colorectal cancer mortality rates were Uruguay, Barbados, Argentina and Cuba, while those with the lowest rates were Guatemala, Canada, Mexico and Honduras. The strongest relationships were found between colorectal cancer mortality rate and the availability of animal fat, red meat, alcoholic beverages and calories. No protective effect of availability of fruits and vegetables on the colorectal cancer mortality rate was found. It would be advisable to improve the records of tumor incidence and direct ways of evaluating diet to be analyzed in future studies instead of the data used here.
\end{abstract}

KEY WORDS Colorectal Neoplasms; Mortality; Diet; Food Supply; Risk Factor. 


\section{INTRODUCCIÓN}

El cáncer colorrectal es un problema de salud pública creciente que afecta cada vez más a los países menos desarrollados. Si bien tiene un componente genético, la influencia del patrón alimentario sobre su desarrollo ha sido ampliamente demostrado. En la medida que los países de América adoptan prácticas alimentarias de los países desarrollados, el crecimiento de las enfermedades crónicas no trasmisibles se hace preocupante.

En ese contexto surgen las siguientes preguntas: ¿cómo influye nuestro patrón alimentario en el desarrollo del cáncer colorrectal?, ¿triplicamos las tasas de mortalidad como triplicamos la recomendación del consumo de carnes?, ¿podemos aplicar sin pensar la recomendación internacional o debemos estudiar qué pasa acá antes de extrapolar recomendaciones? Los países vecinos ¿qué relación muestran en cuanto a consumos alimentarios y la tasa de mortalidad por cáncer colorrectal?

Siguiendo la línea de los estudios tradicionales sobre dieta y cáncer, y en virtud de la información disponible, se decidió analizar la dieta a través de las disponibilidades alimentarias y el cáncer colorrectal, a través de la tasa de mortalidad.

El propósito de este trabajo fue contribuir al conocimiento de la relación entre las disponibilidades alimentarias en países de América y la mortalidad por cáncer colorrectal con un enfoque local considerando que las recomendaciones actuales sobre dieta y cáncer se han realizado estudiando dietas de países de Europa, Asia y América del Norte, principalmente. La investigación se desarrolló en el marco de la tesis titulada "Disponibilidad Alimentaria y mortalidad por cáncer colorrectal en la Argentina y en los demás países de América en el año 2010"(1), para la obtención del título de magíster en Epidemiología, Gestión y Políticas en Salud, en el Instituto de Salud Colectiva, de la Universidad Nacional de Lanús.

\section{Cáncer colorrectal}

El problema del cáncer en el mundo tendrá un impacto creciente en las próximas décadas, como producto del envejecimiento de la población, debido a la mayor expectativa de vida y a la disminución del índice de natalidad observado. El cáncer colorrectal es el tercer tipo de cáncer más frecuente en ambos sexos con tasas de incidencias mundiales del 14,7 por 100.000 habitantes en mujeres y del 20,4 por 100.000 habitantes en hombres. Argentina supera el promedio mundial, con incidencias de cáncer colorrectal para el año 2008 del 16,7 por 100.000 habitantes en mujeres y 25,3 por 100.000 habitantes en hombres ${ }^{(2)}$. Según Gualdrini y lummato ${ }^{(3)}$ :

La sobrevida global del CCR [cáncer colorrectal] es del $60 \%$, y asciende a 90\% cuando es diagnosticado en etapas tempranas. En nuestro país cuando se diagnostica se encuentra en etapas avanzadas [...] La mortalidad por CCR alcanza el $11 \%$ de la mortalidad por tumores malignos, es uno de los tumores con mayor incidencia a nivel mundial. Dos de cada cinco casos de CCR en el mundo en el año 2008 se produjeron en los países menos desarrollados [...] Alrededor del $75 \%$ de los pacientes con CCR son esporádicos, se desarrollan en personas que no presentan antecedentes personales ni hereditarios demostrados de la enfermedad $y$, por lo tanto, factores dietéticos y ambientales han sido implicados en la etiología. En este grupo mayoritario de casos esporádicos, la edad es el principal factor de riesgo [...] La prevención primaria tiene como objetivos identificar los factores de riesgo en la dieta y en el estilo de vida para intentar modificarlos a través de la educación de la población. Tanto la dieta como el estilo de vida, la quimioprevención y los antioxidantes pueden incidir en las diferentes etapas del desarrollo del CCR, ya sea previamente a la aparición de los adenomas, durante el crecimiento de estos o en el proceso de transformación a cáncer ${ }^{(3)}$. 


\section{Dieta como factor de prevención}

La posibilidad de prevenir localizaciones tumorales muy frecuentes a través de cambios en los hábitos alimentarios, especialmente, por el consumo de frutas y verduras (o su considerado equivalente, el suplemento con vitaminas y otros antioxidantes de la dieta) generó, en la década de 1980, fundadas esperanzas para el control del cáncer.

El Instituto Nacional del Cáncer sostiene que, en la medida que factores ambientales como los alimentos, la nutrición y la actividad física inciden en su desarrollo, puede decirse que el cáncer es una enfermedad prevenible.

\begin{abstract}
Así, dietas con alta densidad calórica, elevada proporción de carnes procesadas, alimentos refinados, grasas y alcohol, se asocian a un incremento del riesgo de desarrollar cáncer de mama, próstata y colon, mientras que una alimentación que incluya importantes cantidades de vegetales y frutas, con alto contenido de micronutrientes y fibra, se encuentra relacionada a una disminución del riesgo ${ }^{(4)}$.
\end{abstract}

En el año 2011, un panel de expertos del World Cancer Research Fund (WCRF) elaboró una revisión sobre dieta, actividad física y cáncer colorrectal en el que revisó los estudios al respecto y expidió recomendaciones. En este informe se declara que la dieta juega un papel importante en la prevención y causalidad del cáncer ${ }^{(5)}$.

El informe del WCRF establece, como factor de protección, la actividad física y los alimentos que contienen fibra dietaria (frutas $\mathrm{y}$ verduras) y, como factor de riesgo convincente, aparecen el consumo de carnes rojas, carnes procesadas, las bebidas alcohólicas en hombres, la grasa abdominal y la altura alcanzada en la edad adulta.

Un incremento diario de 100 gramos de carnes rojas aumentaría el riesgo de padecer cáncer colorrectal entre un $12 \%$ y un $17 \%$, y un aumento de 25 gramos en el consumo de carnes procesadas aumentaría el riesgo de cáncer colorrectal en $49 \%{ }^{(5)}$.
Recientemente, la Organización Mundial de la Salud (OMS) publicó un informe de prensa sobre carnes rojas y cáncer en el cual postula:

Después de una revisión exhaustiva de la literatura científica acumulada, un Grupo de Trabajo de 22 expertos de 10 países, convocados por el Programa de Monografías de la IARC [International Agency for Research on Cancer], clasificó el consumo de carne roja como probablemente carcinógeno para los humanos, basado en evidencia limitada de que el consumo de carne roja causa cáncer en los humanos y fuerte evidencia mecanicista apoyando un efecto carcinógeno. Esta asociación se observó principalmente con el cáncer colorrectal, pero también se han visto asociaciones con el cáncer de páncreas y el cáncer de próstata ${ }^{(6)}$.

\section{Formas de medir la dieta}

Las dificultades para acceder a la dieta de las poblaciones han llevado a algunos epidemiólogos a postular que no es posible establecer un perfil dietario poblacional ${ }^{(7)}$. La medición de la ingesta de alimentos en individuos y poblaciones se realiza mediante diversos métodos que difieren tanto en la forma de recoger la información como en el período de tiempo que abarcan. Existe gran controversia sobre cuál de estos métodos es el más adecuado y cuál refleja de manera más fidedigna el consumo real de alimentos de una población. De hecho, diversas revisiones al respecto concluyen que no existe un método enteramente satisfactorio por sí mismo y la utilidad de cada uno dependerá de las condiciones en que se use y de los objetivos con que se realice la medición ${ }^{(8)}$.

La disponibilidad alimentaria representa la cantidad de alimentos disponible por habitante $y$, si bien no refleja diferencias en el acceso a los alimentos ni consumo real, constituye una idea general de la dieta de las poblaciones. Se obtiene a través de las hojas 
de balance de alimentos, las cuales fueron publicados por la Food and Agriculture Organization (FAO) por primera vez en 1949, y se publican sistemáticamente cada año. Las hojas de balance de alimentos son un balance (relación entrada-salida) de alimentos a nivel nacional de la cantidad de un alimento disponible para el consumo humano ${ }^{(9)}$.

\section{Antecedentes de estudios similares}

Los estudios ecológicos de correlación han sido usados tradicionalmente para estudiar dieta y cáncer en las poblaciones ${ }^{(7)}$. Toman las disponibilidades alimentarias de los países para aproximarse a la dieta y las tasas de mortalidad para medir el cáncer. Ambas medidas son poblacionales y, en general, de buena calidad.

\section{MÉTODOS}

Se realizó un estudio ecológico, cuyo objetivo general fue describir la relación entre la disponibilidad alimentaria y la mortalidad por cáncer colorrectal en Argentina y en los demás países de América, en el año 2010.

Se tomaron disponibilidades alimentarias de 33 de 34 países de América (exceptuando Antigua y Barbuda, por falta de datos): Argentina, Bahamas, Barbados, Belice, Bolivia, Brasil, Canadá, Costa Rica, Colombia. Cuba, Chile, Dominica, Ecuador, El Salvador, Estados Unidos, Granada, Guatemala, Guyana, Haití, Honduras, Jamaica, México, Nicaragua, Panamá, Paraguay, Perú, República Dominicana, San Cristóbal y Nieves, San Vicente y Las Granadinas, Santa Lucía, Surinam, Uruguay, Venezuela.

Se estudió la mortalidad por cáncer colorrectal en hombres y mujeres en 2010 de 26 de los 34 países de América (excluyendo a países con datos no confiables): Argentina, Bahamas, Barbados, Bolivia, Brasil, Canadá, Costa Rica, Colombia, Cuba, Chile, Ecuador, El Salvador, Estados Unidos, Guatemala, Guyana, Haití, Honduras, Jamaica, México, Nicaragua, Panamá, Paraguay, Perú, República Dominicana,
Uruguay, Venezuela. Se relacionó la disponibilidad alimentaria con la mortalidad por cáncer colorrectal en estos 26 países.

Para llevar a cabo esta investigación se utilizaron datos provenientes de hojas de balance de alimentos, disponibles en $\mathrm{FAO}^{(10)}, \mathrm{y}$ las tasas de mortalidad por cáncer colorrectal disponibles en la International Agency for Research on Cancer (IARC) ${ }^{(11)}$, para los códigos C18, C19, C20 y C21 de la Clasificación Internacional de Enfermedades (CIE-10).

Se planteó primero una descripción de las disponibilidades alimentarias relacionadas con el cáncer colorrectal: calorías, grasas totales, grasa de origen animal, carne roja, bebidas alcohólicas y frutas y verduras. No se describió la disponibilidad alimentaria de carnes procesadas, a pesar de estar muy relacionadas con el cáncer colorrectal, por carecer del dato en las hojas de balance de alimentos y por no disponer de un dato de buena calidad para ser tomado en cuenta. Luego se describieron las tasas de mortalidad por cáncer colorrectal ajustadas por edad y por grupos quinquenales desde los 55 a los 74 años. Finalmente se relacionó la disponibilidad alimentaria con la tasa de mortalidad por cáncer colorrectal.

\section{Disponibilidad alimentaria}

Para analizar la disponibilidad alimentaria se utilizaron las hojas de balance de alimentos provistas por FAO. Estas proveen información por habitante y por año de kilocalorías, grasas totales, y grasa de origen animal, según país. También brindan información de disponibilidad de alimentos por persona por año. Se comparó la disponibilidad de kilocalorías, grasas totales, grasa de origen animal, carnes rojas, alcohol y frutas y vegetales no feculentos por habitante por país en el año 2010. Estas cantidades fueron expresadas en cantidades diarias para compararlas con las recomendaciones.

Para construir la variable disponibilidad de carnes rojas por habitante, se tomó la definición del WCRF y se procedió a sumar los ítems: carne de bovino, carne de caprino y 
cordero, carne de porcino, otras carnes y vísceras (no se consideró carne de pollo acorde a la definición de carnes rojas) en la hoja de balance de alimentos del país, en el año 2010.

Para analizar la disponibilidad alimentaria de frutas y verduras por habitante, de las hojas de balance se sumó vegetales no feculentos al ítem frutas.

Para construir la variable disponibilidad de alcohol por habitante, se tomó de las hojas de balance el ítem bebidas alcohólicas, se calculó la cantidad por día por habitante de cada bebida y luego se calculó el contenido de etanol aportado por cada bebida. Finalmente se sumaron los contenidos de etanol de cada bebida.

\section{Tasa de mortalidad por cáncer colorrectal}

Se tomaron datos elaborados por la IARC y la $\mathrm{OMS}^{(12)}$. Se utilizó la tasa estandarizada por edad y tasas específicas por edad de 55 a 74 años en grupos quinquenales, con el fin de profundizar el análisis. Se usaron ambos grupos de tasas debido a que aportan datos complementarios.

La tasa estandarizada por edad es una medida resumen de las tasas de mortalidad de una población que considera que todas las poblaciones tienen la misma estructura etaria. La estandarización es necesaria para comparar poblaciones, ya que estas difieren en edad y la edad es un factor influyente en el riesgo de morir por cáncer.

\section{Análisis de correlación}

Para el análisis de las relaciones entre las disponibilidades alimentarias y las tasas de mortalidad por cáncer colorrectal se utilizó el coeficiente de correlación Spearman (rho). Esta medida estadística permite hacer correlaciones cuando existen valores distantes o extremos o cuando la distribución de las variables no responde a una distribución tipo campana de Gauss o normal ${ }^{(13)}$.
Se considera que coeficientes de correlación entre 0 y 0,25 o entre 0 y - 0,25 indican escasa o nula correlación, entre 0,25 y 0,50 (o $-0,25$ a $-0,50)$ indican cierto grado de correlación; de 0,50 a 0,75 o de $-0,50$ a $-0,75)$ correlación moderada y mayor a $0,75(0-0,75)$ es muy buena o excelente. $^{(13)}$

\section{RESULTADOS}

\section{Disponibilidad alimentaria}

La mediana de disponibilidad calórica para la región de las Américas fue de $2.665 \mathrm{kcal}$. (Colombia). El mínimo aporte fue encontrado en Bolivia $(2.168 \mathrm{kcal})$ y el máximo en EEUU (3.659 kcal) seguido de Canadá (3.397 kcal), Brasil (3.230 kcal), Cuba (3.159 kcal) y Argentina ( $3.157 \mathrm{kcal}$ ). La mitad de los países (rango intercuartílico) presentó disponibilidades calóricas entre 2.532 y $3.041 \mathrm{kcal}$ (Tabla 1).

La mediana de disponibilidad de grasas totales fue $85 \mathrm{~g} / \mathrm{hab} . / \mathrm{d}$ á. El valor máximo lo presentó EEUU con $161 \mathrm{~g} / \mathrm{hab} . /$ día seguido de Canadá, Brasil y Argentina (149, 113 y $113 \mathrm{~g} / \mathrm{hab}$./día respectivamente). Los países con las menores disponibilidades de grasa fueron Haití, Perú y Bolivia $(47,47$ y 48 g/ hab./día). El rango intercuartílico fue de 6194 gramos (Tabla1).

Tabla 1. Estadísticos de la variable disponibilidad alimentaria en los países de América, año 2010.

\begin{tabular}{|c|c|c|c|}
\hline Disponibilidad alimentaria & Mediana & $\begin{array}{c}\text { Rango } \\
\text { intercuartílico } \\
\mathrm{Q}_{1} \mathrm{Q}_{3}\end{array}$ & $\begin{array}{c}\text { Coeficiente } \\
\text { de variación } \\
\text { (\%) }\end{array}$ \\
\hline Calórica (kcal/hab./día) & 2.665 & $2.532-3.041$ & 12,8 \\
\hline Grasas totales (g/hab./día) & 85 & $61-94$ & 33,2 \\
\hline Grasa de origen animal (g/hab./día) & 35 & $27-54$ & 42,6 \\
\hline Carne roja (g/hab./día) & 94 & $41-128$ & 55,6 \\
\hline Bebidas alcohólicas (g etanol/hab./día & 10 & $6-12$ & 80,3 \\
\hline Frutas y verduras ( $\mathrm{g} / \mathrm{hab} . / \mathrm{dí}$ ) & 421 & $341-538$ & 39,2 \\
\hline
\end{tabular}


Los países con mayores cantidades de grasa animal disponible fueron EEUU, $\mathrm{Ar}$ gentina, Canadá y Las Bahamas $(69,68,67$ y $63 \mathrm{~g} / \mathrm{hab} . /$ día respectivamente). Los países con menor disponibilidad fueron Haití, Perú y Guatemala (10, 16 y 17 g/hab./día respectivamente). La mediana fue de $35 \mathrm{~g} / \mathrm{hab} . / \mathrm{d}$ ía y el rango intercuartílico 27-54 (Tabla 2).

La mediana del porcentaje de kcal aportadas por grasas totales fue de 26 , con un coeficiente de variación del $22 \%$ y un rango intercuartílico de 22-31. El mínimo valor fue encontrado en Perú con un aporte calórico proveniente de grasas del $16 \%$ y el máximo en EEUU, donde las grasas totales aportaron el $40 \%$ del valor calórico. Para las calorías provenientes de grasa de fuente animal la mediana fue de $13 \mathrm{~g} / \mathrm{hab} . /$ día con un coeficiente de variación del 33\% (rango intercuartílico 10-16). Los países en el extremo superior (EEUU, Argentina, Canadá y Bahamas) presentaron un consumo aparente cinco veces mayor que los países en el extremo inferior (Haití y Perú) (Figura 1).

Al analizar las tres variables concernientes a disponibilidad alimentaria general (disponibilidad calórica, de grasas totales y de grasa animal), se pudo ver que la distribución de la disponibilidad calórica presentó menos variaciones (coeficiente de variación: $12,8 \%$ ) que la distribución de la disponibilidad de grasas totales (coeficiente de variación: $33,2 \%$ ) y que la distribución de grasa de origen animal (coeficiente de variación: 42,6\%) (Tabla 1).

La disponibilidad de carnes rojas mostró una gran variabilidad en los países estudiados con un coeficiente de variación del 55,6\%. La mediana fue de $94 \mathrm{~g} / \mathrm{hab} . /$ día. La Tabla 2 muestra que los países con los niveles más altos fueron Argentina (194g), EEUU (190g), Uruguay (162g), Canadá (155g), Bahamas (150g) y Brasil (148g). Los países con menores disponibilidades fueron San Cristóbal y Nieves (15g), Guyana (19g), Nicaragua (29g) y Haití (32g) (Tabla 2). El rango intercuartílico fue de 41-128 (Tabla 1).

La disponibilidad media de alcohol (medida en gramos de etanol) mostró gran diferencia entre países. El país con mayor disponibilidad (EEUU: 20 g/etanol/día) presentó casi siete veces más disponibilidad que el último (Guatemala: 3 g/etanol/día) (Figura 2).

El consumo aparente de frutas y vegetales no feculentos presentó una fluctuación importante entre países, con un coeficiente de variación del 39,2\%. Los países con mayor disponibilidad (Dominica y Bahamas) presentaron siete veces más disponibilidad que el país en el rango inferior (Nicaragua); aunque, en general, la disponibilidad fue baja para la mayoría de los países. Apenas siete países alcanzaron la disponibilidad plena de futas y vegetales (660 gramos, esto es la recomendación más 10\%); Dominica, Bahamas, San Vicente y las Granadinas, Belice, Cuba y República Dominicana y Canadá, y solo un país, EEUU, tuvo disponibilidad suficiente (600 gramos, 100\%) (Figura 3).

\section{Tasa de mortalidad por cáncer colorrectal}

Las tasas de mortalidad por cáncer colorrectal estandarizadas mostraron un comportamiento similar a las específicas por edad de 55 a 74 años con gran variabilidad entre los países, con un coeficiente de variación mayor al $40 \%$ en todos los grupos estudiados.

Uruguay presentó la tasa más alta de mortalidad por cáncer colorrectal, en todos los grupos. Otros países que presentaron tasas considerablemente más altas que el resto fueron Bahamas, Cuba, y EEUU. Barbados presentó tasas altas en casi todos los grupos estudiados, excepto en el grupo de 55 a 59 años. Argentina se ubicó en tercer lugar en la tasa de mortalidad por cáncer colorrectal estandarizada y entre el sexto y séptimo en las tasas específicas de 55 a 74 años. Los países con las tasas más bajas en todos los quinquenios estudiados fueron Guatemala, Canadá, México y Haití (Figura 4).

La tasa de mortalidad por cáncer colorrectal ajustada por edad presentó una mediana de 6,5 por 100.000 habitantes y un rango intercuartílico de 5,4-8,8. Los países con mayores tasas fueron: Uruguay (16 por 
Tabla 2. Disponibilidad alimentaria diaria por habitante, según país. América, 2010.

\begin{tabular}{|c|c|c|c|c|c|c|}
\hline País & $\begin{array}{l}\text { Calorías } \\
\text { (kcal/día) }\end{array}$ & $\begin{array}{l}\text { Grasas } \\
\text { totales } \\
\text { (g/día) }\end{array}$ & $\begin{array}{l}\text { Grasa } \\
\text { animal } \\
\text { (g/dia) }\end{array}$ & $\begin{array}{l}\text { Frutas y } \\
\text { vegetales } \\
\text { (g/dia) }\end{array}$ & $\begin{array}{l}\text { Carne roja } \\
\text { (g/día) }\end{array}$ & $\begin{array}{l}\text { Etanol } \\
\text { (g/dia) }\end{array}$ \\
\hline Argentina & 3.157 & 113 & 68 & 388 & 194 & 13 \\
\hline Bahamas & 2.584 & 95 & 63 & 1005 & 150 & 15 \\
\hline Barbados & 3.060 & 94 & 51 & 538 & 89 & 9 \\
\hline Belice & 2.839 & 73 & 41 & 815 & 60 & 11 \\
\hline Bolivia & 2.168 & 48 & 33 & 284 & 94 & 4 \\
\hline Brasil & 3.230 & 113 & 54 & 501 & 148 & 12 \\
\hline Canadá & 3.397 & 149 & 67 & 671 & 155 & 12 \\
\hline Chile & 2.949 & 85 & 54 & 368 & 128 & 11 \\
\hline Colombia & 2.665 & 78 & 32 & 461 & 68 & 7 \\
\hline Costa Rica & 2.869 & 87 & 38 & 435 & 81 & 9 \\
\hline Cuba & 3.159 & 64 & 33 & 739 & 98 & 9 \\
\hline Dominica & 3.069 & 85 & 47 & 1119 & 83 & 10 \\
\hline Ecuador & 2.450 & 100 & 52 & 558 & 96 & 4 \\
\hline El Salvador & 2.524 & 58 & 27 & 341 & 36 & 5 \\
\hline EEUU & 3.659 & 161 & 69 & 604 & 190 & 20 \\
\hline Granada & 2.451 & 95 & 37 & 470 & 51 & 11 \\
\hline Guatemala & 2.431 & 57 & 17 & 362 & 34 & 3 \\
\hline Guyana & 2.623 & 54 & 24 & 317 & 19 & 6 \\
\hline Haití & 2.169 & 47 & 10 & 240 & 32 & 9 \\
\hline Honduras & 2.573 & 71 & 27 & 352 & 41 & 5 \\
\hline Jamaica & 2.762 & 79 & 34 & 513 & 36 & 9 \\
\hline México & 3.041 & 91 & 45 & 421 & 103 & 7 \\
\hline Nicaragua & 2.512 & 61 & 22 & 158 & 29 & 4 \\
\hline Panamá & 2.579 & 72 & 35 & 317 & 101 & 11 \\
\hline Paraguay & 2.575 & 93 & 38 & 366 & 113 & 9 \\
\hline Perú & 2.617 & 47 & 16 & 474 & 42 & 8 \\
\hline República Dominicana & 2.532 & 88 & 29 & 730 & 68 & 10 \\
\hline San Cristóbal y Nieves & 2.499 & 84 & 42 & 263 & 15 & 8 \\
\hline San Vicente y las Granadinas & 2.944 & 83 & 42 & 980 & 74 & 12 \\
\hline Santa Lucía & 2.640 & 78 & 55 & 356 & 101 & 13 \\
\hline Surinam & 2.804 & 81 & 27 & 423 & 61 & 10 \\
\hline Uruguay & 2.955 & 88 & 54 & 384 & 162 & 12 \\
\hline Venezuela & 2.906 & 88 & 36 & 340 & 103 & 13 \\
\hline
\end{tabular}

100.000.), Barbados (14 por 100.000) y Argentina (13 por 100.000) Los países con valores inferiores fueron: Guatemala y Canadá (3 por 100.000) y México (4 por 100.000).

Al analizar la tasa discriminada por edad en la franja de 55 a 74 años se observaron aumentos abruptos a medida que aumentaba la edad. La mediana tuvo un valor de 12,5 por 100.000 en la franja de 55 a 59 años a 21,5 por 100.000 en el quinquenio siguiente, luego 31 por 100.000 en la franja de 65 a 69 años, para llegar a 59 por 100.000 en el grupo de 70 a 74 años. 


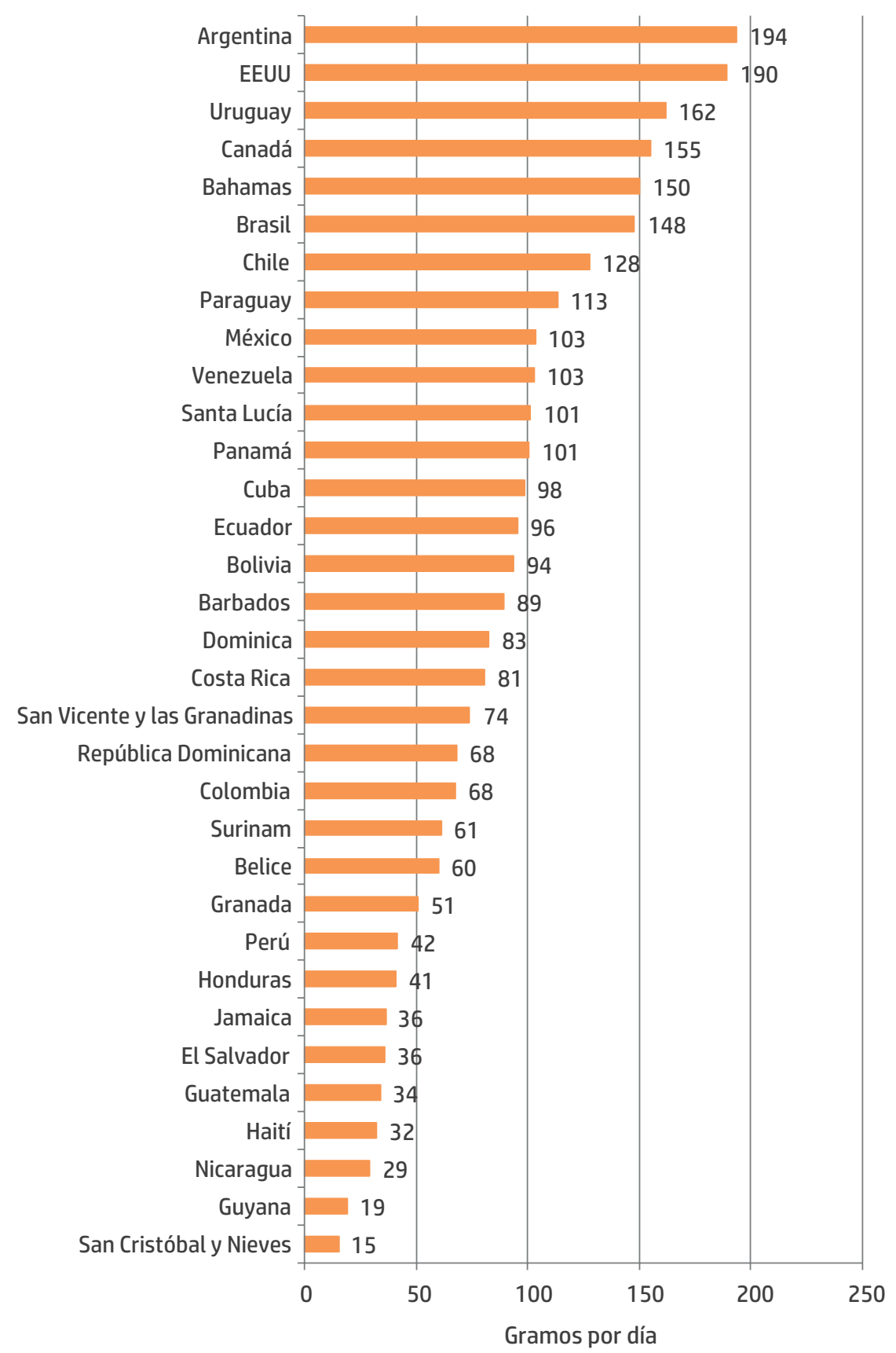

Figura 1. Disponibilidad diaria de carne roja por habitante, según país. América, 2010.

Fuente: Elaboración propia con base en datos de Food and Agriculture Organization ${ }^{(10)}$ 

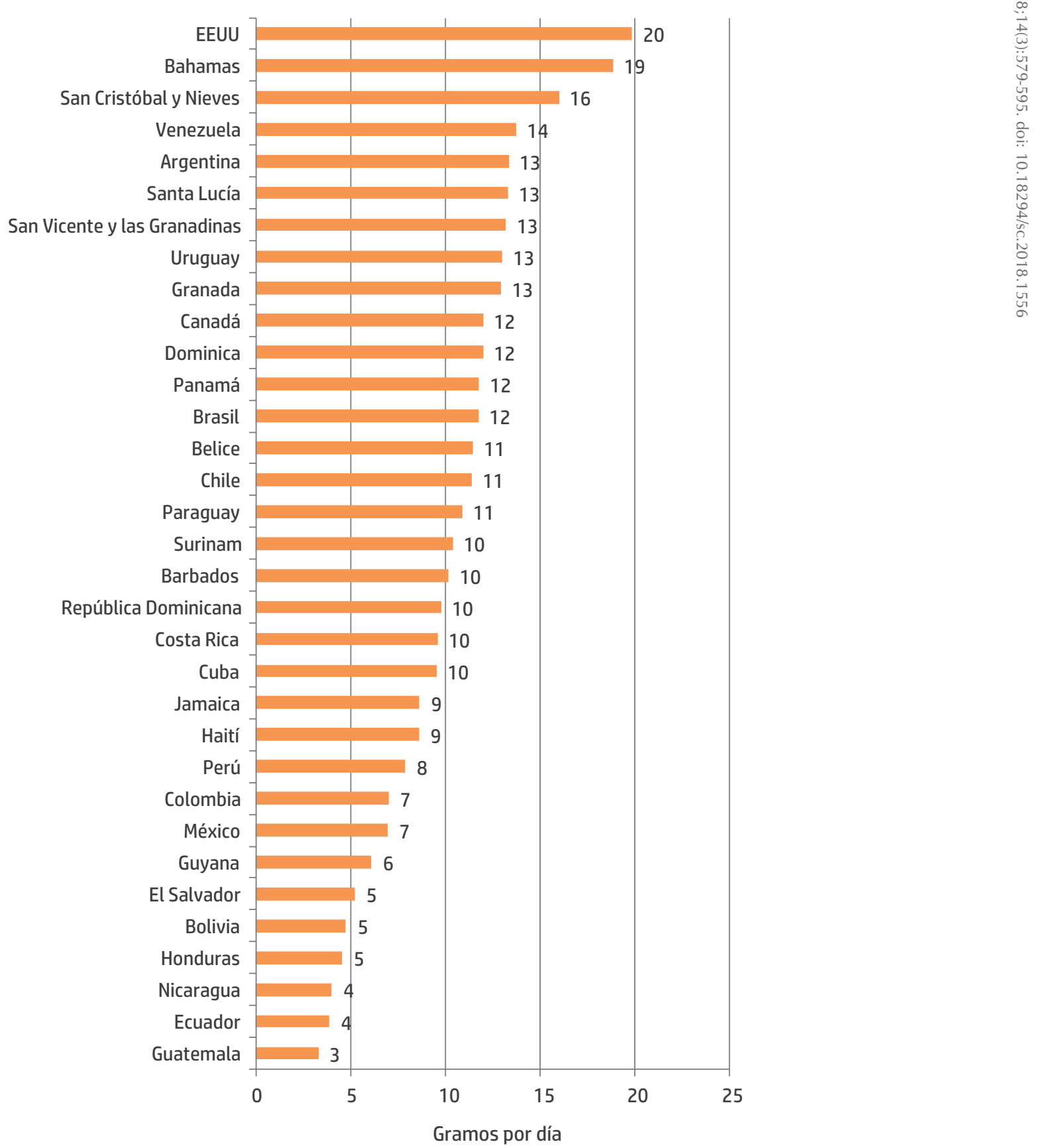

Figura 2. Disponibilidad diaria de bebidas alcohólicas (gramos de etanol) por habitante, según país. América, 2010.

Fuente: Elaboración propia con base en datos de Food and Agriculture Organization ${ }^{(10)}$. 


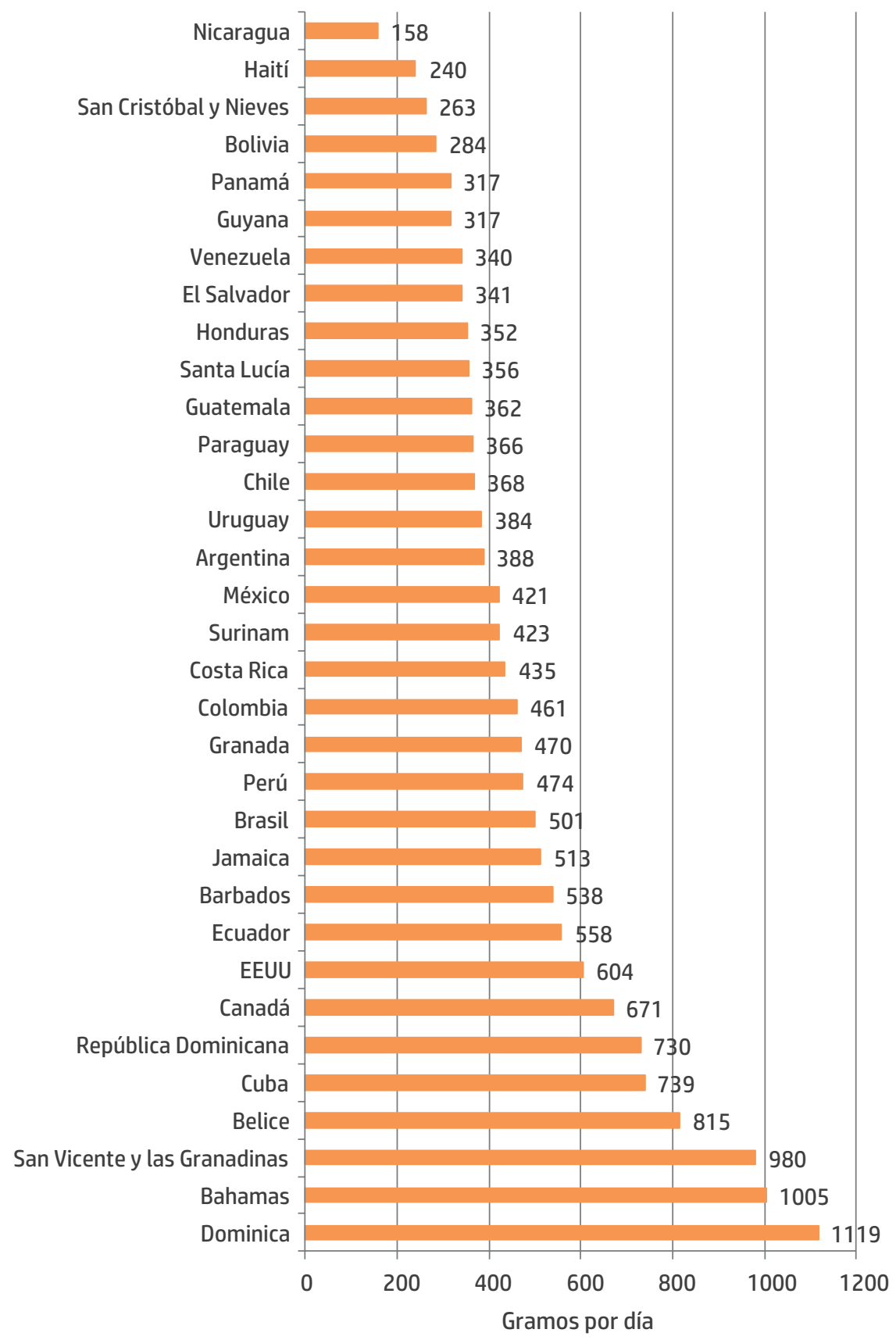

Figura 3. Disponibilidad diaria de frutas y vegetales no feculentos por habitante, según país. América, 2010.

Fuente: Elaboración propia con base en datos de Food and Agriculture Organization ${ }^{(10)}$. 


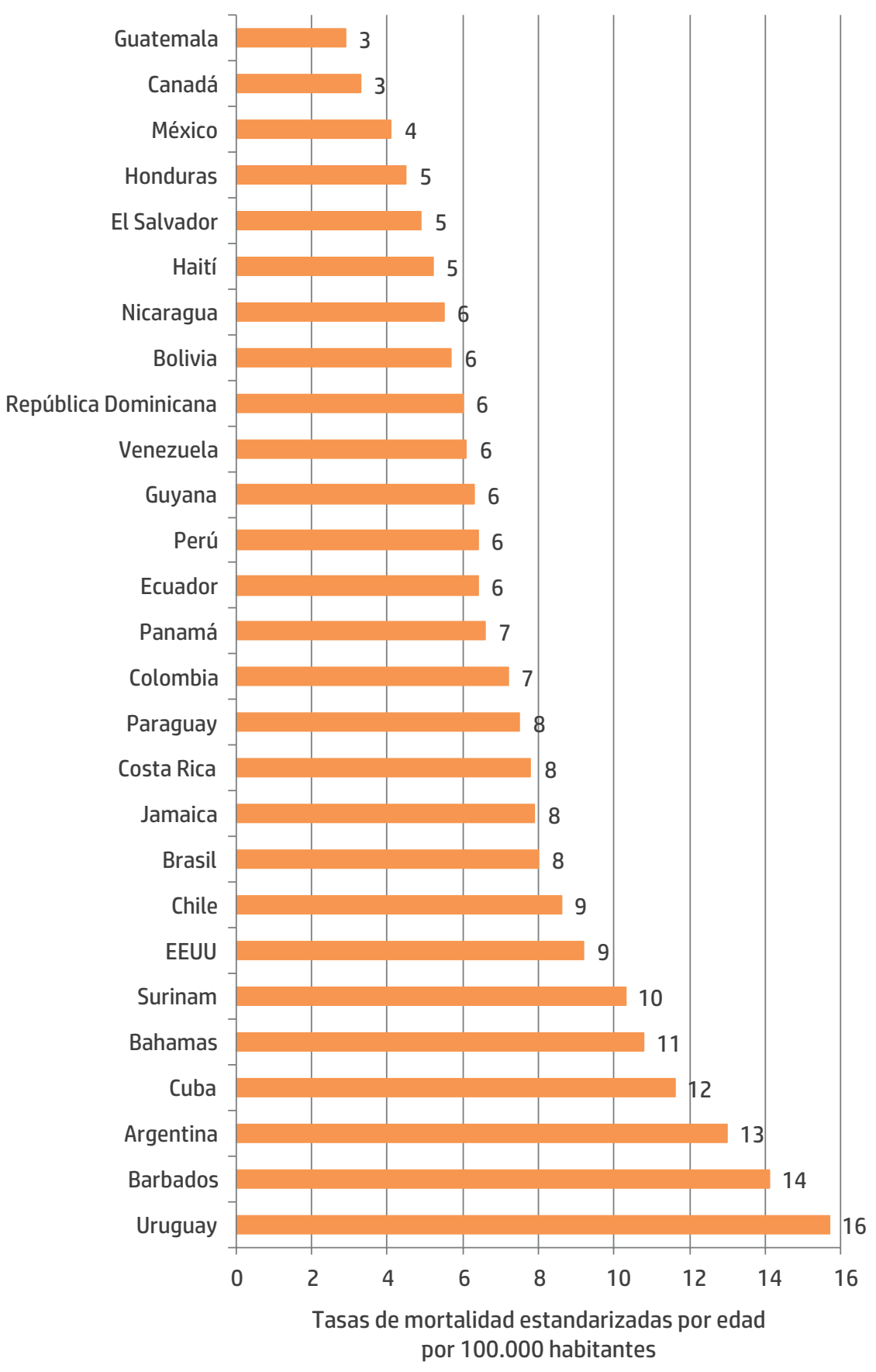

Figura 4. Tasas de mortalidad estandarizadas por edad de cáncer colorrectal (por 100.000 habitantes), según país. América, 2010.

Fuente: Elaboración propia con base en International Agency for Research on Cancer $^{(11)}$. 


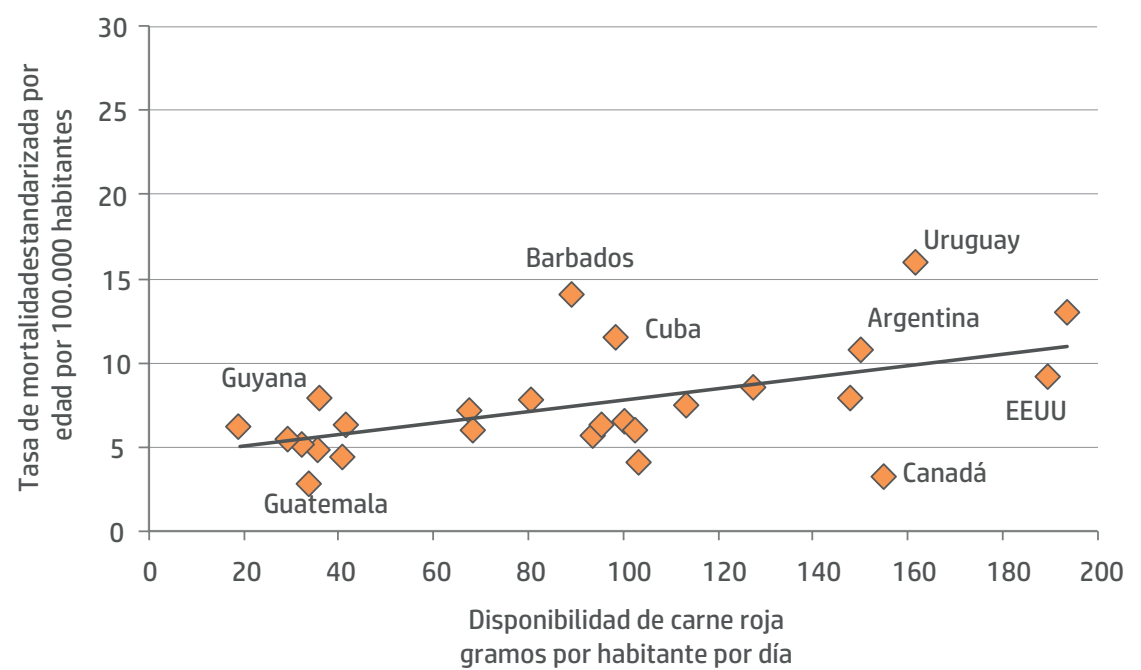

Figura 5. Relación entre la disponibilidad de carne roja y la tasa de mortalidad estandarizada por edad de cáncer colorrectal (por 100.000 habitantes), según país. América, 2010.

Fuente: Elaboración propia con base en Food and Agriculture Organization ${ }^{(10)}$ y en International Agency for Research on Cancer ${ }^{(11)}$

\section{Relaciones entre disponibilidad alimentaria y mortalidad por cáncer colorrectal}

Se analizó la relación entre la disponibilidad de carne roja y la tasa de mortalidad por cáncer colorrectal estandarizada por edad. Se encontró una asociación moderada positiva con un coeficiente de correlación de Spearman de 0,59 y un coeficiente de determinación de 0,29 . Se observaron países con alta disponibilidad de carnes rojas y alta tasa de mortalidad por cáncer colorrectal estandarizada como Uruguay y Argentina, países con disponibilidades medias y altas tasas como Barbados y Cuba y países con altas disponibilidades de carnes rojas y tasas bajas o medias como Canadá y EEUU (Figura 5).

Al relacionar la disponibilidad de etanol y la tasa de mortalidad por cáncer colorrectal estandarizada por edad se obtuvo un coeficiente de correlación de Spearman de 0,61 , esto es una relación positiva moderada. La mayoría de los países con disponibilidades altas de alcohol, presentaron tasas altas también. Sin embargo, EEUU, el país con mayor disponibilidad, presentó una tasa intermedia y Canadá, con disponibilidad alta, presentó una de las tasas más bajas.

La relación entre la disponibilidad de frutas y verduras no feculentas y la tasa de mortalidad por cáncer colorrectal manifestó una correlación débil positiva. Lo anterior contradice las recomendaciones que dan, a este grupo de alimentos, un rol protector.

La relación entre disponibilidad calórica y la tasa de mortalidad por cáncer colorrectal estandarizada por edad fue moderada positiva con un coeficiente de correlación de Spearman de 0,56 y un coeficiente de determinación de 0,16.

La relación entre la disponibilidad de grasas totales y la tasa de mortalidad por cáncer colorrectal fue débil positiva (rho: 0,47). Los países con mayor disponibilidad de grasa, EEUU y Canadá, presentaron tasas de mortalidad por cáncer colorrectal intermedias y bajas, respectivamente. Luego, países como Uruguay, Barbados y Argentina presentaron disponibilidades de grasa intermedias y tasas altas.

Por último, se encontró una relación un poco más fuerte para la disponibilidad de 
grasa de fuente animal y la tasa de mortalidad por cáncer colorrectal, con un coeficiente de correlación de Spearman de 0,6 (relación moderada positiva).

\section{DISCUSIÓN Y ANÁLISIS}

\section{Disponibilidad alimentaria}

Los resultados encontrados con respecto a la disponibilidad alimentaria mostraron una gran variabilidad entre los países. Las diferencias fueron mayores en disponibilidad de alimentos que en aporte calórico. Se pudo observar un cierto perfil dentro de cada país. Los países con mayor disponibilidad calórica, tuvieron también mayor disponibilidad de grasas totales, de grasas de origen animal, de alcohol y de carnes rojas. La disponibilidad de frutas y verduras mostró menos variación entre los países que los demás alimentos estudiados.

A primera vista aparecen los países más desarrollados y los grandes productores de alimentos con las mayores disponibilidades y los más pobres con las disponibilidades más bajas. Aun los países con disponibilidades menores alcanzaron niveles de suficiencia para las kilocalorías lo que coincide con lo postulado por la FAO y con el aumento de la obesidad ${ }^{(14)}$.

Los países con mayores cantidades de grasas totales disponibles tuvieron un alto porcentaje de calorías provistas por grasas (entre $31 \%$ y $40 \%$ del valor calórico total) y una alta disponibilidad calórica, a excepción de Ecuador y Granada que presentaron alta disponibilidad de grasas y baja disponibilidad calórica. Los países con menores disponibilidades de grasas totales fueron Haití, Perú, Bolivia y Guyana, todos con menos de 55 gramos de grasa/hab./día y con aproximadamente $20 \%$ del valor calórico total aportado por grasas.

Dentro de los países con mayor disponibilidad de grasa animal se encuentran EEUU, Argentina, Canadá y Santa Lucía con disponibilidad de grasa animal superior a la disponibilidad de grasas totales de los países en el límite inferior. Cinco países (Perú, Haití,
Bolivia, Guyana, Guatemala y El Salvador) presentaron disponibilidades de grasa animal menores a $25 \mathrm{~g} / \mathrm{hab} . /$ día.

Lo anterior coincide con lo expresado en la bibliografía respecto a la convivencia del hambre con la obesidad, y a un número considerable de personas que padecen hambre, a pesar de que la prevalencia global de desnutrición haya disminuido ${ }^{(14)}$.

Si se compara la disponibilidad de carne roja antes comentada con la recomendación de ingesta para prevenir cáncer colorrectal (máximo de 70 gramos de carne roja al día) se puede observar que la disponibilidad de la mayoría de los países de América es muy superior (Figura 1). Dieciséis países superan ampliamente la disponibilidad plena para carnes rojas. Dentro de estos Ilaman la atención Argentina y EEUU con disponibilidades cercanas a los 190 g/hab./día y, en segunda línea, Uruguay, Canadá, Bahamas y Brasil, con disponibilidades cercanas a los $150 \mathrm{~g} / \mathrm{hab}$./día.

\footnotetext{
Particularmente en Argentina, el perfil alimentario tradicional está caracterizado por un alto consumo de proteínas y grasas animales, obtenidas principalmente de las carnes rojas, y una baja ingesta de pescado, frutas y verduras. Además, es habitual en la región el asado de carnes a la parrilla. ${ }^{(4)}$
}

Solo nueve países presentaron disponibilidades de carne roja menores a $50 \mathrm{~g} / \mathrm{hab}$./ día (San Cristóbal y Nieves, Guyana, Nicaragua, Haití, Guatemala, El Salvador, Jamaica, Honduras y Perú). Estos países presentaron también disponibilidades calóricas bajas (a excepción de Jamaica). Lo que podría estar indicando que la baja disponibilidad de carne roja respondería a una baja disponibilidad alimentaria general.

La disponibilidad de bebidas alcohólicas presentó una variabilidad del $43 \%$. Las proporciones de etanol en las bebidas son muy variables y esta conversión puede no ser exacta. Los límites de ingesta de alcohol sugeridos para prevenir enfermedades varían según sexo y están dirigidas a adultos. La disponibilidad de bebidas alcohólicas 
está expresada en las hojas de balance por habitante, sin discriminar edad ni sexo. El consumo de alcohol se da preferentemente en adultos y adolescentes y discrimina por sexo siendo, en general, más alto en hombres que en mujeres ${ }^{(15)}$. Por todo lo expuesto anteriormente, de todos los componentes de la disponibilidad alimentaria, el alcohol es el más difícil de comparar con las recomendaciones, pero sí es pertinente la comparación entre países, ya que las limitaciones se dan por igual en todos los países.

La disponibilidad de frutas y vegetales no feculentos fue baja en general con una mediana de $421 \mathrm{~g} / \mathrm{hab} . /$ día, muy por debajo de las recomendaciones de IARC de 600 g/día.

Esto nos hace reflexionar sobre la dificultad de los individuos, en el resto de los países, de cumplir con la recomendación, particularmente, los grupos menos favorecidos, en los cuales el acceso a alimentos suele estar comprometido. Sería interesante analizar las tendencias temporales de disponibilidad de frutas y vegetales para discernir si se trata de una recomendación desproporcionada a la capacidad histórica de proveer alimentos de los países o si se debe a una coyuntura actual. En este último caso, vale preguntarse: ¿qué está pasando que tantos países no alcanzan una buena disponibilidad de frutas y vegetales?, ¿se cultiva menos?, ¿se cultiva con fines no alimentarios?

Con referencia a los fines no alimentarios de los cultivos se ha postulado que "los biocombustibles pueden ser también una amenaza a la seguridad alimentaria, al destinar tierras aptas para cultivos a la producción de cereales y semillas con fines energéticos"(16).

\section{Análisis de la mortalidad por cáncer colorrectal}

Se observó una gran variabilidad en las tasas de mortalidad por cáncer colorrectal, tanto en la tasa estandarizada por edad como en las tasas quinquenales de 55 a 74 años con un coeficiente de variación mayor al $40 \%$ en todos los grupos estudiados. Uruguay lideró todos los grupos de mortalidad. Otros países con alta mortalidad fueron Barbados, Argentina, Cuba, EEUU y Bahamas.

Llama la atención que entre los países con más altas tasas encontramos países como EEUU y Bahamas con economías estables y producto bruto interno de 53.000 y 31.500 dólares per cápita, respectivamente, y la presencia de Argentina, Uruguay y Barbados con PBI de 15.000 dólares per cápita, aproximadamente, y Cuba con un PBI de 6.000 dólares per cápita.

Los países con menores tasas de mortalidad por cáncer colorrectal fueron: Guatemala, Canadá, México, Haití y Honduras. Se destaca la presencia de Canadá, país con un producto bruto interno (PBI) de 52.000 per cápita, con alta incidencia de cáncer colorrectal pero baja mortalidad, donde estarían operando el diagnóstico precoz y tratamiento oportuno como factores que disminuyen la mortalidad. Los demás países con tasas bajas fueron países con $\mathrm{PBI}$ menores 4.000 dólares per cápita, a excepción de México país en vías de desarrollo con un PBI de 10.000 dólares per cápita.

\section{Análisis de las relaciones entre disponibilidad alimentaria y tasas de mortalidad por cáncer colorrectal}

Se encontraron relaciones positivas moderadas entre la tasa de mortalidad por cáncer colorrectal y la disponibilidad calórica de grasa de fuente animal, de carnes rojas y de alcohol. El panel de expertos de OMS manifiesta una relación poco convincente entre el consumo de grasa de origen animal y cáncer colorrectal ${ }^{(17)}$. En este estudio la relación fue positiva débil.

El reporte del 2011 sobre cáncer colorrectal y dieta del WCRF tampoco hace recomendaciones sobre grasas en la dieta ${ }^{(5)}$. En este estudio, se encontró relación positiva débil entre la tasa de mortalidad por cáncer colorrectal y la disponibilidad de grasas totales, lo que coincide con la bibliografía actual.

Con respecto a la carne roja, un estudio ecológico clásico y pionero realizado por Armstrong y Doll en 1975 encontró una correlación de 0,85 para los hombres y 0,89 
para mujeres ${ }^{(7)}$, a diferencia de la correlación de 0,59 para ambos sexos aquí encontrada.

\section{Análisis de las fortalezas y limitaciones de este estudio}

Los estudios tradicionales de dieta y cáncer han sido básicamente estudios ecológicos de correlación en los que se comparaba la tasa de mortalidad y dieta en países centrales. La fuente de datos para cuantificar la dieta ha sido la disponibilidad alimentaria.

En ese sentido, este estudio replica la técnica comparando los datos en los países de América abarcando países al norte y sur del Ecuador.

El rol de los estudios ecológicos en la epidemiología nutricional es controversial. Por un lado, han estimulado gran parte de la investigación en cáncer y dieta y enfatizado en la diferencia en las tasas de mortalidad entre países. También han sido considerados como fuente pobre para determinar causalidad por sus múltiples efectos de confusión y por las dificultades para medir la dieta en forma directa ${ }^{(18)}$.

Como en todo estudio ecológico, se debe tener cuidado de no cometer falacia ecológica, inferir a nivel individual a partir del nivel grupal. Los resultados y recomendaciones deben hacerse en el mismo nivel que fueron tomados los datos, esto es el nivel poblacional.

\section{RECOMENDACIONES}

Existen aproximaciones más exactas al cáncer colorrectal y a la dieta que no fueron utilizadas por no contar con datos de buena calidad en la mayoría de los países estudiados.

En cuanto al cáncer colorrectal la incidencia es una medida mucho más exacta que la mortalidad. Esta última se ve afectada por el diagnóstico precoz y el tratamiento oportuno, el cual difiere entre los países y no depende de la dieta. En este sentido, el diagnóstico y el tratamiento pueden ser considerados factores de confusión en este estudio ya que países con alta incidencia y buen diagnóstico y tratamiento van a presentar baja mortalidad, como en el caso de Canadá. Lamentablemente también hay mucha diferencia en la calidad del dato de incidencia entre los países desarrollados y subdesarrollados.

En América Latina y el Caribe hay carencia de registros de gran calidad. De acuerdo con el volumen IX de la publicación de IARC Cancer incidence in five continents 2007, solo el $6 \%$ de la población está cubierta por registros de cáncer con base poblacional, en contraste con el $83 \%$ en América del Norte y $32 \%$ en Europa ${ }^{(19)}$.

La disponibilidad alimentaria, como forma de caracterizar la dieta, si bien ha sido muy usada, tiene algunas limitaciones importantes: no diferencia por sexo, edad ni condiciones especiales, y no muestra la disponibilidad de productos alimentarios. Hace cincuenta años esto no era relevante, pero hoy en día gran parte de la alimentación se basa en alimentos ultra procesados. De esta manera, las carnes procesadas no han sido tomadas en este estudio por carecer del dato.

Según el panel de expertos de la OMS sobre dieta y enfermedades crónicas, para comprender mejor la relación entre las características del consumo de alimentos, las dietas y la aparición de enfermedades no transmisibles, es crucial obtener información más fidedigna sobre las pautas y tendencias reales del consumo de alimentos, basada en estudios representativos del consumo ${ }^{(20)}$. Es fundamental utilizar una forma directa de medir la dieta en poblaciones que discrimine no solo por tipo de alimentos base, sino que pueda diferenciar a los alimentos procesados. Para superar lo anterior estudios europeos sobre dieta y cáncer han usado otras metodologías $y$, en base a los datos de estos estudios, se formularon las recomendaciones del WCRF usadas en este trabajo ${ }^{(5,6,7,7,9,9,12,13,14,17)}$.

El European prospective investigation into cancer and nutrition (EPIC) es uno de los estudios de cohorte más grandes del mundo con 521 millones de participantes de 10 países, seguidos durante 15 años. Viene 
utilizando una combinación de métodos para medir la dieta de las poblaciones. Al utilizar una combinación de métodos se diluyen las limitaciones de cada método por separado.

Si mejoraran los registros de incidencia de cáncer y se sistematizara una forma de medir la dieta en los países de América se podría acceder a información fehaciente que permitiera relacionar la dieta y el cáncer colorrectal en la región de una forma más exacta. Del mismo modo, el registro de incidencia de cáncer y de dieta en forma directa permitiría hacer asociaciones con otras variables de interés que podrían estar operando en este fenómeno de salud enfermedad como el nivel socioeconómico, sexo, obesidad, actividad física, etc.

\section{CONCLUSIONES}

Existe una gran variabilidad en la disponibilidad alimentaria en los países de América y en las tasas de mortalidad por cáncer colorrectal. La disponibilidad calórica, de grasas totales, de grasa de fuente animal y de carnes rojas es plena en la mayoría de los países, los que no alcanzan la disponibilidad suficiente de frutas y verduras para cumplir con las recomendaciones del IARC, lo que merece ser estudiado en profundidad, dado que este grupo de alimentos se considera fundamental en una alimentación saludable.

Entre los países con mayores tasas de mortalidad por cáncer colorrectal se encontraron países de ingresos medios y altos. Se encontraron relaciones positivas moderadas entre la tasa de mortalidad por cáncer colorrectal y la disponibilidad calórica, de grasa animal, bebidas alcohólicas y carne roja. Lo que coincide con estudios anteriores, pero con distinta fuerza. La relación encontrada con la grasa de origen animal fue más fuerte en este estudio que lo postulado por la bibliografía; para las demás variables la relación fue más débil que lo postulado anteriormente. Se encontró una relación positiva débil entre la tasa de mortalidad por cáncer colorrectal y la disponibilidad de grasa y la disponibilidad de frutas y vegetales no feculentos, a diferencia de las recomendaciones que sugerirían un efecto protector.

Sería recomendable mejorar los registros de incidencia de tumores para ser tomados en futuros estudios en lugar de los registros de mortalidad. También sería aconsejable sistematizar formas directas de medir la dieta para una aproximación más exacta a las dietas de las poblaciones. Si se pudieran generalizar registros de obesidad y actividad física a nivel país, sería muy útil incluirlos en futuros estudios, así como el nivel socioeconómico de los países.

\section{AGRADECIMIENTOS}

A Adrián Santoro, director de mi tesis de maestría y guía en esta investigación.

\section{REFERENCIAS BIBLIOGRÁFICAS}

1. Buamden S. Disponibilidad Alimentaria y mortalidad por cáncer colorrectal en la Argentina y en los demás países de América en el año 2010. [Tesis de maestría]. Lanús: UNLa; 2017.

2. Instituto Nacional de Cáncer, Ministerio de Salud de la de la Nación. Boletínes [Internet]. Instituto Nacional de Cáncer; 2011 [citado 15 may 2015]. Disponible en: https://tinyurl.com/ yсрspmj8.

3. Gualdrini UA, lummato LE. Cáncer colorrectal en la Argentina: organización, cobertura y calidad de las acciones de prevención y control; Informe final de diciembre de 2011: diagnóstico de situación de la Argentina. Buenos Aires: Instituto Nacional de Cáncer; 2011.

4. Pou SA, Niclis C, Aballay LR, Tumas N, Román MD, Muñoz SE, Becaria Coquet J, Díaz MP. Cáncer y su asociación con patrones alimentarios en Córdoba, Argentina. Nutrición Hospitalaria. 2014;29(3):618-628.

5. World Cancer Research Fund. Continuous update project colorectal cancer 2011 report: food, nutrition, physical activity and the prevention of the colorectal cancer. Washington DC: American Institute for Cancer Research, World Cancer Research Fund; 2011.

6. Agencia Internacional de Investigación sobre el Cáncer, Organización Mundial de la Salud. Comunicado de prensa $N^{\circ}$ 240: monografías de la 
IARC evalúan el consumo de la carne roja y de la carne procesada [Internet]. Lyon: Organización Mundial de la Salud; 2015 [citado 1 ago 2017]. Disponible en: https://tinyurl.com/yctj2ct4.

7. Willett W. Nutritional epidemiology. New York: Oxford University Press; 2013.

8. Cabuli SD. Metodologías para calcular el consumo de alimentos de en una población. Revista del Hospital JM Ramos Mejía. 2007;12(3):1-8.

9. Cotier JP, Morón C. Hojas de balance de alimentos. En: Morón C, Zacarías I, De Pablo S. Producción y manejo de datos de composición química de alimentos en nutrición [Internet]. Santiago de Chile: Food and Agriculture Organization; 1997 [citado 1 ago 2017]. Disponible en: https:// tinyurl.com/yc87o7uj.

10. Food and Agriculture Organization. Food Balance Sheet [Internet]. 2017 [citado 1 ago 2017]. Disponible en: https://tinyurl.com/hubuj39.

11. Ferlay J, Soerjomataram I, Ervik M, Dikshit R, Eser S, Mathers C, Rebelo M, Parkin DM, Forman D, Bray F, (eds.). GLOBOCAN 2012: Estimated Cancer Incidence, Mortality and Prevalence Worldwide in 2012 v1.0. Lyon: IARC; 2012.

12. Ferlay J, Soerjomataram I, Ervik M, Dikshit R, Eser S, Mathers C, Rebelo M, Parkin DM, Forman D, Bray F, (edits.). GLOBOCAN 2012: Estimated Cancer Incidence, Mortality and Prevalence worldwide in 2012 [Internet]. Lyon: International Agency for Research on Cancer; 2013 [citado 1 ago 2017]. Disponible en: http://gco.iarc.fr/today/home.

13. Dawson Saunders B, Trapp R. Bioestadística médica. México DF: El Manual Moderno; 1997.
14. Organización de las Naciones Unidas para la Alimentación y la Agricultura. El Estado de la inseguridad alimentaria en el mundo 2013: las múltiples dimensiones de la seguridad alimentaria [Internet]. Roma: Food and Agriculture Organization; 2013 [citado 1 ago 2017]. Disponible en: http://www.fao.org/3/a-i3434s.pdf.

15. World Cancer Research Fund. Alimentos, nutrición, actividad física y la prevención del cáncer: una perspectiva mundial [Internet]. Washington DC: World Cancer Research Fund, American Institute for Cancer Research; 2007 [citado 1 ago 2017]. Disponible en: https://tinyurl.com/y8fdfy3k.

16. Organización Panamericana de la Salud. Informe sobre la situación regional sobre el alcohol y la salud en las Américas [Internet]. Washington DC: Organización Panamericana de la Salud; 2015 [citado 1 ago 2017]. Disponible en: https:// tinyurl.com/ya6bq7uh.

17. Bravo Velásquez E. Encendiendo el debate sobre biocombustibles: cultivos energéticos y soberanía alimentaria en América Latina. Buenos Aires: Capital Intelectual; 2007.

18. Kinlen LJ. Fat and cancer. British Medical Jo urnal.1983;286(6371):1081-1082.

19. Piñeros M. La iniciativa mundial para el desarrollo de registros de cáncer (GICR): una herramienta para el desarrollo de capacidades en el registro de cáncer [Internet]. Lyon: International Agency for Research on Cancer; 2014 [citado 1 ago 2017]. Disponible en: https://tinyurl.com/y794s5qc.

20. Organización Mundial de la Salud. Dieta, nutrientes y enfermedades crónicas (Informe Técnico 916). Ginebra: OMS; 2003. 